\title{
Avaliação do impacto da prematuridade na saúde mental de puérperas
}

\author{
Manina deSaza FilhoFavaro- CentroUnivesitániodeAraraquara, Araraquara, SãoPaulo, Brasil \\ Rodingsannhes Peres - UniversidadeFeekral deUtbelândia, Uberlândia, Minas Gerais, Brasil \\ Manod Antôniodos Santos- UniversidadedeSão Paulo Riberão Preto, SãoPaulo, Brasil
}

\begin{abstract}
Resumo
O presente estudo tem como objetivo comparar a ocorrência de sintomas de ansiedade e depressão em mães de bebês prematuros e mães de bebês a termo. Trata-se de um estudo transversal, descritivo, de abordagem quantitativa. As participantes $(n=40)$ foram submetidas à avaliação de rastreamento executada como rotina pelo Serviço de Psicologia de um hospital filantrópico do interior paulista mediante a aplicação da Escala Hospitalar de Ansiedade e D epressão (HAD ). Constatou-se que, entre as mães de bebês prematuros, $75 \%$ apresentavam sintomas clinicamente significativos de ansiedade e $50 \%$ apresentavam sintomas clinicamente significativos de depressão. Já entre as mães de bebês a termo, $65 \%$ não apresentavam sintomas clinicamente significativos de ansiedade e tampouco depressão. Ademais, a superioridade da pontuação média obtida pelas primeiras alcançou significância estatística. Os resultados corroboram a literatura, que sugere que a prematuridade tende a ter impacto negativo na saúde mental da mulher que vivencia essa situação.

Palavasdhave D epressão; Ansiedade; Nascimento prematuro; Psicologia hospitalar.
\end{abstract}

Evaluation of the impact of prematurity in mental health in puepenium

\begin{abstract}
The present study intends to compare the occurrences of anxiety and depression symptoms in mothers of premature infants and in mothers of term infants. It is a quantitative descriptive and transversal study. The sample $(\mathrm{n}=40)$ underwent screening assessment carried out as routine by the Psychology Service of a philanthropic hospital in the state of São Paulo with Hospital Anxiety and Depression Scale (HAD). Among mothers of premature infants, $75 \%$ showed clinically meaningful symptoms of anxiety and 50\% showed clinically meaningful symptoms of depression. However, among mothers of term infants, $65 \%$ didn't showed clinically meaningful symptoms of anxiety or depression. Moreover, the superior average score obtained by the mothers of premature infants reached statistical significance. Therefore, the results reinforce, as suggested by the specialized scientific literature, that prematurity tends to have a negative impact on the health of a woman going through such situation.

Kegwards D epression; Anxiety; Premature birth; Hospital psychology.
\end{abstract}

Evaluación del impacto de la prematuridad en la salud mental de puérperas

\section{Resumen}

El presente estudio tiene como objetivo comparar la ocurrencia de síntomas de ansiedad y depresión en madres de bebés prematuros y madres de bebés nacidos a término. Se trata de un estudio transversal, descriptivo, de abordaje cuantitativa. Las participantes $(n=40)$ fueron sometidas a la evaluación de rastreamento ejecutada como rutina por el Servicio de Psicología de un hospital filantrópico del interior del estado de São Paulo-Brasil mediante la aplicación de la Escala Hospitalaria de Ansiedad y D epresión (HAD ). Se constató que, entre las madres de bebés prematuros, un 75\% presentaba síntomas clínicamente significativos de ansiedad y un 50\% presentaba síntomas clínicamente significativos de depresión. Ya entre las madres de bebés nacidos a término, un 65\% no presentaba síntomas clínicamente significativos de ansiedad y tampoco depresión. A demás, la superioridad del puntaje medio obtenido por las primeras alcanzó significancia estadística. Los resultados corroboran la literatura, que sugiere que la prematuridad tiende a tener impacto negativo en la salud mental de la mujer que vivencia esa situación.

Palabras dave D epresión; Ansiedad; Nacimiento prematuro; Psicología hospitalaria.

\section{Gestação e prematuridade: aspectos psicológicos}

O parto prematuro - ou seja, aquele que ocorre com menos de 37 semanas completas de gestação tende a frustrar as expectativas relacionadas ao nascimento do bebê, de modo que pode ser considerado um acontecimento potencialmente desestruturante para a família e, principalmente, para a mãe. Tal fato é compreensível, sobretudo, porque a condição biológica do bebê geralmente torna a internação em uma Unidade de Terapia Intensiva Neonatal (UTIN) um imperativo para sua sobrevivência (Moreira \& Rodrigues, 2003). Logo, muitas mães que passam por essa experiência apresentam, conforme Carvalho, Linhares, Padovani e Martinez (2009), diversos sentimentos negativos, tais como: apreensão resultante da incerteza da evolução clínica do bebê, tristeza desencadeada pela separação precoce imposta pela internação e culpa por não ter conseguido conduzir a gestação até o final.

A mãe também cabe a árdua tarefa de se adaptar emocionalmente à "imagem real" do bebê, abrindo mão da "imagem ideal" alimentada ao longo da gravidez por fantasias de natureza inconsciente (Kennell \& Klaus, 1993). Bebês prematuros geralmente são pequeninos, apresentam baixo peso e têm a pele amarelada ou escurecida. Nos casos em que a adaptação matema a essa realidade não é bemsucedida, a mãe, muitas vezes, se toma incapaz de perceber que as diferenças que separam seu filho de um bebê a termo podem ser apenas temporárias. Isso tende a dificultar a formação de um vínculo afetivo consistente entre a díade e comprometer a qualidade dos cuidados maternos (Klein \& Linhares, 2006; Wilfong, Saylor \& Elksnin, 1991). 
Vale destacar, ainda, que não raro mães de bebês prematuros evitam se apegar com 0 intuito de minimizar um eventual sofrimento emocional posterior (Raad, Cruz \& Nascimento, 2006). Tal fato acontece porque alguns bebês prematuros, principalmente aqueles que correspondem à classificação de "muito baixo peso" (inferior a $1.500 \mathrm{~g}$ ) ou "extremo baixo peso" (inferior a $1.000 \mathrm{~g}$ ), correm risco de morte relacionado à imaturidade dos órgãos internos e sistemas corporais. De modo geral, quanto menor a idade gestacional e o peso ao nascimento, pior 0 prognóstico. Segundo Moreira e Rodrigues (2003), os problemas de saúde mais frequentes nesse contexto são: apneia, anemia, displasia broncopulmonar, hemorragia intraventricular, asfixia, pneumomediastino e taquipneia transitória. Porém, a gravidade desses problemas varia caso a caso.

\section{Repencussões psicológicas da hospitalização neonatal}

A primeira visita materna à UTIN gera uma mistura de emoções intensas. 0 cenário formado por tecnologia médica ostensiva, a quantidade de aparelhos e a movimentação dos profissionais causa perplexidade e enseja uma estranha sensação de irrealidade. 0 contato com o bebê se torna, assim, mais laborioso (Raad \& cols., 2006). 0 estabelecimento de um vínculo inconsistente entre a mãe e seu bebê prematuro, contudo, pode, de acordo com diversos autores, potencializar as chances do mesmo vir a apresentar problemas neurológicos, prejuízos sensoriais, desordens motoras, dificuldades cognitivas, distúrbios comportamentais e transtornos afetivos durante a infância ou adolescência (Carvalho, Linhares \& Martinez, 2001; Vieira \& Linhares, 2011).

Além disso, existem evidências empíricas de que a hospitalização do bebê prematuro pode desencadear na mãe diferentes tipos de transtornos afetivos. Em um estudo nacional, por exemplo, Padovani, Linhares, Carvalho, Duarte e Martinez (2004) investigaram, mediante a aplicação de uma bateria de testes psicológicos, a ocorrência de sintomas maternos de ansiedade, disforia e depressão em dois momentos distintos: durante e após a internação do bebê em UTIN. Na primeira avaliação, $44 \%$ das participantes $(\mathrm{n}=19)$ apresentaram sintomatologia clinicamente significativa de, ao menos, um desses quadros clínicos. Já na segunda avaliação, esse número se reduziu para $26 \% \quad(\mathrm{n}=11)$, a despeito do desenvolvimento de intervenções. Os autores, assim, destacam a importância da assistência psicológica preventiva junto a essa população.

O nascimento prematuro do bebê, portanto, é capaz de tornar a mulher particularmente suscetível aos transtornos mentais específicos do puerpério, ou seja, do período que se segue do parto até o retorno dos órgãos genitais e do estado geral da mulher à normalidade. A depressão pós-parto é um desses transtornos, conforme Cantilino, Zambaldi, Sougey e Rennó Junior (2010), a prematuridade é um de seus principais fatores de risco. Além disso, a mãe deprimida se mostra incapaz de apresentar um adequado nível tanto de atividade quanto de responsividade (Wijnroks, 2000), assim como de cuidar de seu bebê de forma lúdica e de sustentar contato visual frequente (Schwengber \& Piccinini, 2003), o que tende a prejudicar o desenvolvimento infantil em termos emocionais e sociais (Schmidt, Picolotto \& Müller, 2005).

Transtornos ansiosos também podem ocorrer com frequência no puerpério (Cantilino \& cols., 2010), particularmente em mães de bebês prematuros (Pinelli, 2000). Como se sabe, a ansiedade é um estado emocional normal em circunstâncias adversas. Porém, sua manifestação se torna patológica quando é desproporcional às circunstâncias que a desencadeiam (D algalarrondo, 2008). A incerteza da evolução clínica tende a precipitar expectativa apreensiva na população em questão, uma vez que a sensação de perigo iminente à saúde do bebê geralmente não corresponde ao risco real (Padovani, Carvalho, Duarte, Martinez \& Linhares, 2009). Essa expectativa, assim, se converte em experiências subjetivas incômodas e estados corporais desagradáveis correlativos da hiperatividade do sistema nervoso autonômico.

Evidencia-se, tendo em vista o que precede, a necessidade de uma atenção especial à saúde mental de mães de bebês prematuros, sobretudo durante a hospitalização neonatal. Porém, ainda são escassos os estudos que tratam dessa temática. Novos estudos são importantes, inclusive, para que se possa identificar instrumentos com boa relação custo-benefício capazes de serem utilizados para fins de rastreamento nesse contexto. Desse modo, 0 presente estudo foi desenvolvido com o objetivo de avaliar e comparar a ocorrência de sintomas de ansiedade e depressão em mães de bebês prematuros internados para tratamento em UTIN e em mães de bebês a termo internados para observação pós-parto em maternidade.

\section{Deserhomtadlóg்co}

\section{Método}

0 presente estudo adotou 0 desenho metodológico da pesquisa documental, priorizando um caráter descritivo, retrospectivo e com abordagem quantitativa. Ademais, se alinha ao enfoque de pesquisa naturalística, uma vez que não tem como propósito principal testar hipóteses, mas sim descrever um fenômeno tal como ele naturalmente ocorre e nas circunstâncias que espontaneamente 0 geram (Selltiz, Wrightsman \& Cook, 1987).

\section{Amotra}

A amostra foi composta por 40 puérperas, sendo 20 mães de bebês prematuros (Grupo 1) e 20 mães de 
bebês a termo (Grupo 2). A Tabela 1 apresenta uma caracterização sociodemográfica das participantes, quanto à idade, estado civil, escolaridade e ocupação. Já na Tabela 2, se encontra uma caracterização clínica das mães, considerando-se as seguintes variáveis: número de filhos, idade gestacional do bebê e tempo de internação. Observa-se, assim, que o perfil das participantes é mais semelhante em relação à ocupação e ao estado civil. Vale destacar que se trata de uma amostra não-probabilística de conveniência, na medida em que foi constituída por puérperas consideradas elegíveis por terem sido submetidas às avaliações de rastreamento executadas pelo Serviço de Psicologia de um hospital filantrópico paulista no período de agosto a dezembro de 2006.

Tabela 1. Caracterização sociodemográfica das puérperas avaliadas

\begin{tabular}{llcc}
\hline Características sociodemográficas & Grupo 1 & Grupo 2 \\
\hline Idade materna (média / variação) & 26,75 anos $(19-42)$ & 24,7 anos (18 - 35) \\
\hline Estado civil & Solteiras & 4 & 4 \\
& Casadas & 12 & 10 \\
& Amasiadas & 4 & 6 \\
\multirow{2}{*}{ Escolaridade } & Ensino fundamental incompleto & 1 & - \\
& Ensino fundamental completo & 4 & 8 \\
& Ensino médio incompleto & 5 & 3 \\
& Ensino médio completo & 8 & 6 \\
& Ensino superior incompleto & 1 & 1 \\
& Ensino superior completo & 1 & 2 \\
\hline Ocupação atual & Donas de casa & 8 & 9 \\
& Profissões de nível fundamental & 3 & 3 \\
& Profissões de nível médio & 6 & 6 \\
& Profissões de nível superior & 1 & 2 \\
& Estudantes & 1 & - \\
\hline
\end{tabular}

Foram estabelecidos os seguintes critérios de exclusão: mães que apresentavam antecedentes psiquiátricos relatados, suspeita de déficit intelectual, quadros demenciais ou distúrbios da comunicação que pudessem comprometer a interação verbal. Com base nos critérios de inclusão/exclusão, dentre as 58 puérperas que constituíram a amostra inicial do estudo, 18 foram excluídas. Vale destacar ainda que, no hospital em questão, após se recuperarem do parto, as mães de bebês prematuros recebem alta e os neonatos seguem internados na UTIN, ao passo que as mães dos bebês a termo permanecem internadas em alojamento conjunto com os neonatos para observação pós-parto, recebendo alta por volta de dois dias após 0 nascimento.

Tabela 2. Caracterização clínica das puérperas avaliadas

\begin{tabular}{lcc}
\hline Características clínicas & Grupo 1 & G rupo 2 \\
\hline Número de filhos (média / variação) & 2,1 filhos (1 - 5) & 1,7 filhos (1 - 4) \\
Idade gestacional do bebê (média / variação) & 31,8 semanas $(24$ - 36) & 39,85 semanas (37 - 42) \\
Tempo de internação (média / variação) & 11,85 dias (2 - 45) & 1,5 dias (1 - 2) \\
\hline
\end{tabular}

\section{Instrumento}

$\mathrm{O}$ instrumento adotado no presente estudo foi a Escala Hospitalar de Ansiedade e D epressão (HAD), a qual tem sido amplamente utilizada no mundo todo para a avaliação de sintomas clinicamente significativos de ansiedade e depressão em adultos de ambos os sexos. Não se trata, portanto, de um instrumento de diagnóstico, mas sim de rastreamento. Compõem a HAD 14 itens do tipo múltipla escolha, os quais se dividem em uma subescala de 7 itens para depressão (HAD-D) e outra subescala de 7 itens para ansiedade (HAD-A). 0 escore global das subescalas varia de $0 \mathrm{a}$ 21 pontos e é resultado da pontuação total obtida em cada um dos itens, com gradação de 0 a 3 (Zigmund \& Snaith, 1983).
A versão em português da HAD já foi testada e validada em diferentes populações no contexto de saúde. Botega, Bio, Zomignani, Garcia Júnior e Pereira (1995), por exemplo, mensuraram a consistência interna da HAD-A e a HAD-D em uma amostra de pacientes internados por condições clínicas diversas em uma enfermaria geral de adultos. O s coeficientes alfa de Cronbach obtidos foram, respectivamente, de 0,68 e 0,77 . Além disso, os coeficientes de validação atingidos com o ponto de corte 8/9 foram, em termos de sensibilidade e especificidade, de $93,7 \%$ e $72,6 \%$ para a avaliação de sintomas de ansiedade, e de $84,6 \%$ e 90,3\% para a avaliação de sintomas de depressão.

Em um estudo com pacientes ambulatoriais portadores de dor crônica, Castro e colaboradores 
(2006), por sua vez, reportaram os seguintes resultados quanto à validação do instrumento em questão: para a HAD-A, 91,7\% de sensibilidade e 41,8\% de especificidade, ao passo que, para a HAD-D , 73,3\% de sensibilidade e $67,2 \%$ de especificidade, ambos com 0 ponto de corte 8/9. Vale mencionar ainda que Marcolino e colaboradores (2007) verificaram, com uma amostra de pacientes internados com indicação para procedimentos cirúrgicos diversos, que a correlação avaliada por meio do coeficiente de correlação de Spearman, entre a HAD-A e o Inventário de Ansiedade de Beck (BAI) e da HAD-D com o Inventário de D epressão de Beck (BDI), variou de 0,68 a 0,70 .

Conclui-se, portanto, que a versão nacional da HAD apresenta indicadores psicométricos semelhantes àqueles frequentemente encontrados em instrumentos de avaliação disponíveis no contexto nacional. Mas é preciso salientar que, em contraste com outros instrumentos que se propõem a objetivos análogos, dentre os quais se pode citar o BDI e o BAI, a HAD não investiga a ocorrência de sintomas vegetativos de ansiedade e depressão, tais como insônia, fadiga e perda de peso (Andrade \& Gorenstein, 2000; Calil \& Pires, 2000). D esse modo, mostra-se especialmente útil conforme Botega e colaboradores (1995), para avaliação de transtornos de humor em indivíduos que apresentam condições clínicas capazes de desencadear sintomas desse tipo. Ademais, segundo Delfini, Roque e Peres (2009), a HAD apresenta como vantagem adicional 0 fato de ser concisa e de aplicação rápida e simples.

Vale mencionar a existência de um instrumento de rastreamento desenvolvido especificamente para puérperas: a Escala de Depressão Pós-Natal de Edimburgo (EPDS). Constituído por 10 itens do tipo múltipla escolha com gradação de 0 a 3, o referido instrumento, tal como a HAD, é conciso e de aplicação rápida e simples (Cox \& Holden, 2003). Da mesma forma, se encontra validado e adaptado em diversos países, inclusive no Brasil, apresentando indicadores psicométricos satisfatórios (Figueira, Corrêa, MalloyDiniz \& Romano-Silva, 2009). A HAD, não obstante, foi selecionada para os fins do presente estudo porque se afigura como um instrumento mais abrangente, já que a EPDS, como seu próprio nome indica, privilegia a avaliação de sintomas de depressão, não contemplando, assim, os sintomas de ansiedade que, como já mencionado, muitas vezes ocorrem em mães de bebês prematuros.

\section{Cdea dedados}

A coleta de dados do presente estudo foi realizada a partir de consultas ao arquivo do Serviço de Psicologia da instituição hospitalar. Constam desse arquivo informações a respeito dos indivíduos (pacientes ou familiares) submetidos às avaliações de rastreamento realizadas como rotina pelo Serviço de
Psicologia. D entre as informações disponíveis, foram registradas mais especificamente aquelas referentes à identificação pessoal e à condição psicológica das puérperas que compuseram o Grupo 1 e o Grupo 2. A identificação pessoal contempla, no caso, as seguintes informações obtidas originalmente junto ao prontuário médico: idade, estado civil, ocupação atual, escolaridade, número de filhos, idade gestacional do bebê e tempo de internação do neonato. Os dados sobre a condição psicológica são aqueles decorrentes da aplicação da HAD, instrumento que faz parte do protocolo de avaliação adotado pelo Serviço de Psicologia do hospital.

Ressalte-se que as avaliações de rastreamento realizadas como rotina pelo Serviço de Psicologia respeitaram uma sequência previamente definida. Cada indivíduo foi abordado individualmente no leito em que se encontrava acomodado. Estabeleceu-se o rappat e forneceram-se os esclarecimentos sobre os objetivos da avaliação. 0 paciente, então, era informado de que os dados coletados seriam utilizados em prol de sua própria assistência no contexto hospitalar, bem como poderiam ser empregados no desenvolvimento de pesquisas científicas, as quais manteriam sua identidade em anonimato. A avaliação seguia somente quando 0 indivíduo consentia com tais condições.

\section{Análisededados}

Em um primeiro momento, foram calculados os escores médios obtidos pelas participantes do Grupo 1 e do Grupo 2 em cada uma das subescalas que constituem a HAD. Em um segundo momento, a diferença entre esses escores médios foi avaliada mediante 0 emprego do Teste t de Student. Foi adotado 0 nível de significância de 5\% para todas as análises realizadas. Em um terceiro momento, foram calculados os escores médios referentes tanto a HAD -A quanto à HAD-D obtidos por cada grupo em função das variáveis sociodemográficas e clínicas consideradas para os fins do presente estudo. Por fim, em um quarto momento, esse conjunto de dados foi discutido em um movimento de articulação com a literatura científica especializada.

\section{Cuidados éticos}

O presente estudo foi desenvolvido com a anuência da direção clínica do hospital em questão e foi aprovado pelo Comitê de Ética em Pesquisa da instituição de filiação da primeira autora quando de sua realização (Protocolo no 631/07), 0 que atesta 0 atendimento às diretrizes propostas pela legislação vigente no país.

\section{Resultados}

Como se pode observar na Tabela 3, foi encontrada significância estatística entre a diferença da pontuação média obtida pelas mães de bebês 
prematuros e pelas mães de bebês a termo tanto na HAD -A quanto na HAD-D. De acordo com a Tabela 4, $75 \%$ das participantes do Grupo $1 \quad(n=15)$ apresentavam sintomas clinicamente significativos de ansiedade. Os itens da HAD-A nos quais se observaram as maiores médias foram os seguintes: 7
(1,90 pontos), 5 (1,90 pontos) e 3 (1,85 pontos). Portanto, os sintomas de ansiedade prevalentes entre as mães de bebês prematuros foram incapacidade de relaxamento, preocupações difusas e expectativas apreensivas.

Tabela 3. Distribuição dos escores médios obtidos nas subescalas da HAD

\begin{tabular}{lccc}
\hline Subescalas & Pontuação - Média - Grupo 1 & Pontuação - Média - Grupo 2 & p $^{*}$ \\
\hline HAD -A & 10,50 & 6,10 & $-2,99$ \\
HAD-D & 8,70 & 5,15 & $-2,72$ \\
\hline
\end{tabular}

* nível de significância $\mathrm{p}<0,05$

A Tabela 4 ainda revela que $50 \%$ das participantes do Grupo $1 \quad(\mathrm{n}=10)$ apresentavam sintomas clinicamente significativos de depressão. Os itens que compõem a HAD-D nos quais ocorreram as maiores médias foram os seguintes: 6 (1,80 pontos) e
14 (1,65 pontos). Isso aponta que os sintomas de depressão experimentados com maior intensidade pelas mães de bebês prematuros avaliadas foram anedonia e incapacidade de engajamento em atividades de lazer.

Tabela 4. Distribuição da classificação dos escores brutos obtidos por subescalas da HAD

\begin{tabular}{llcc}
\hline Sub-escalas & Classificação & Grupo 1 (f) & G rupo 2 (f) \\
\hline \multirow{3}{*}{ HAD-A } & Escores clinicamente significativos (>8) & 15 & 5 \\
& Escore subclínico (8) & 1 & 2 \\
& Escores sem significância clínica $(<8)$ & 4 & 13 \\
\multirow{3}{*}{ HAD -D } & Escores clinicamente significativos $(>8)$ & 10 & 3 \\
& Escore subclínico (8) & 2 & 4 \\
& Escores sem significância clínica $(<8)$ & 8 & 13 \\
\hline
\end{tabular}

Em contraste com o que ocorreu no Grupo 1, $65 \%$ das mães de bebês a termo $(\mathrm{n}=13)$ apresentava sintomas de ansiedade e depressão sem significância clínica. Ainda assim, vale destacar que o sintoma de ansiedade prevalente no Grupo 2 foi tensão inespecífica, dado que no item 1 obteve-se a maior média (1,25 pontos) entre os itens que compõem a HAD-A. Por outro lado, a incapacidade de engajamento em atividades de lazer se sobressaiu enquanto sintoma de depressão para as mães de bebês a termo, na medida em que a média registrada no item 14 (1,15 pontos) foi a mais elevada entre aqueles que compõem a HAD -D.

Tendo-se em vista as características sociodemográficas e clínicas das participantes do Grupo 1 que apresentaram sintomas clinicamente significativos de ansiedade, constatou-se que as maiores médias na HAD-A foram obtidas pelas solteiras $(14,5$ pontos), primíparas (13,6 pontos), com idade entre $31 \mathrm{e}$ 35 anos (14,5 pontos), que possuíam ensino fundamental completo (14,33 pontos), exerciam atividades profissionais de nível médio (15 pontos), completaram 31 semanas de gestação (15 pontos) e cujos bebês somavam de 1 a 5 dias de intemação na UTIN (14,40 pontos).

0 perfil sociodemográfico e clínico das participantes do grupo em questão nas quais foram identificados sintomas clinicamente significativos de depressão é semelhante quanto à idade, escolaridade e ocupação. Porém, vale destacar que atingiram as maiores médias na HAD-D as mulheres amasiadas (13,5 pontos) e multíparas (13 pontos) que completaram 35 semanas de gestação (14 pontos) e cujos bebês se encontravam internados na UTIN por um período que variava de 11 a 15 dias (16 pontos).

No que diz respeito às características sociodemográficas e clínicas das participantes do Grupo 2 que apresentaram sintomas clinicamente significativos de ansiedade, deve-se salientar que atingiram as maiores médias na HAD-A as puérperas casadas (13,5 pontos), mães de 1 ou 2 filhos (14 pontos), com idade entre 26 a 30 anos (15 pontos) e ensino fundamental completo (14 pontos) ou ensino médio incompleto (14 pontos), que exerciam atividades profissionais de nível fundamental (15 pontos), completaram 41 semanas de gestação (16 pontos) e cujos bebês somavam de 1 a 5 dias de internação na maternidade (13,2 pontos).

Por fim, cumpre assinalar que, em conformidade com esses resultados, é possível afirmar que, considerando as características sociodemográficas e clínicas das participantes do grupo em questão que apresentaram sintomas clinicamente significativos de depressão, as maiores médias na HAD-D ocorreram entre puérperas solteiras (14 pontos), primíparas (14 pontos), donas de casa (14 pontos), com idade entre 21 a 25 anos (14 pontos) e ensino médio incompleto (13 pontos), que completaram 39 semanas de gestação (12 pontos) e cujos bebês somavam de 1 a 5 dias de internação na maternidade (11,66 pontos). 


\section{Discussão}

0 presente estudo evidenciou que, entre as participantes do Grupo 1, 75\% ( $\mathrm{n}=15)$ apresentavam sintomas clinicamente significativos de ansiedade, ao passo que $50 \% \quad(\mathrm{n}=10)$ manifestavam sintomas clinicamente significativos de depressão. Os estudos de Padovani e colaboradores (2004) e Padovani e colaboradores (2009), também desenvolvidos no país, reportam resultados distintos em termos proporcionais. Afinal, o primeiro estudo aponta que, em uma amostra de 43 mães de bebês prematuros, foram identificados sintomas clinicamente significativos de ansiedade e depressão em, respectivamente, $35 \%(\mathrm{n}=15)$ e $26 \%$ (n=11) dos casos durante a hospitalização neonatal. Já no segundo estudo, desenvolvido com 50 participantes, a porcentagem de mães de bebês prematuros que apresentaram os referidos sintomas foi ainda mais reduzida: $32 \%(\mathrm{n}=16)$ e $14 \%(\mathrm{n}=7)$.

0 estudo de Carvalho, Linhares, Padovani e Martinez (2009), se comparado com o presente estudo, também reporta a uma menor ocorrência proporcional de sintomas clinicamente significativos de ansiedade e depressão em uma amostra de 59 mães de bebês prematuros. As autoras dividiram aleatoriamente as participantes em dois grupos, em razão do tipo de assistência psicológica oferecida. No Grupo 1, constituído por 36 mulheres que frequentaram um grupo de apoio desenvolvido com o emprego de material informativo, foram identificados sintomas clinicamente significativos tanto de ansiedade quanto de depressão em 39\% dos casos (n=14) antes e em $22 \%$ dos casos $(\mathrm{n}=8)$ após a intervenção. 0 mesmo ocorreu em 30\% ( $\mathrm{n}=7)$ e $9 \%(\mathrm{n}=2)$ das 23 mulheres que compuseram o Grupo 2 e frequentaram um grupo de apoio desenvolvido sem o emprego de material informativo, antes e após, respectivamente, a intervenção.

D eve-se esclarecer que, nos referidos estudos os instrumentos utilizados foram 0 Inventário de Ansiedade Traço-Estado (IDATE) e o BDI, o que pode explicar a diferença dos resultados obtidos em comparação com aqueles reportados no presente estudo. O IDATE avalia, sobretudo, sintomas inespecíficos, tais como astenia, culpabilidade e agitação, que podem ocorrer em qualquer situação de estresse, e 0 segundo claramente privilegia os aspectos cognitivos dos quadros depressivos, os quais incluem desesperança, desamparo e perda de insight (Andrade \& Gorenstein, 2000; Calil \& Pires, 2000). Já a HAD, conforme Botega e colaboradores (1995), enfatiza a sintomatologia psíquica de ansiedade e depressão, 0 que a torna especialmente útil para avaliação de indivíduos que, como as participantes tanto do Grupo 1 quanto do G rupo 2, devido ao puerpério, apresentam condições clínicas capazes de desencadear sintomas físicos análogos aos que ocorrem em quadros depressivos e ansiosos.
Assim como foi observado no estudo de Padovani e colaboradores (2009), o Grupo 2 funcionou, no presente estudo, como grupo controle. Mas 0 fato de terem sido registradas entre as participantes do Grupo 1 médias maiores, com significância estatística, do que entre as participantes do Grupo 2, tanto na HAD-A quanto na HAD-D, obviamente não é um achado inesperado, uma vez que, como já mencionado, diversos autores destacam que 0 nascimento prematuro é um acontecimento potencialmente desestruturante para a mulher que vivencia essa situação (Kennell \& Klaus, 1993; Raad \& cols., 2006). Entretanto, no estudo de Padovani e colaboradores (2009) não houve significância estatística quanto aos sintomas de depressão nos dois grupos avaliados.

A ocorrência de sintomas clinicamente significativos de ansiedade e depressão em uma parcela expressiva das participantes do Grupo 1 deve ser entendida como um indício da necessidade de oferecer assistência psicológica às mães de bebês prematuros durante 0 período de hospitalização neonatal. Nessa direção, estudo realizado por Pinelli (2000) com mais de uma centena de participantes atestou que níveis elevados de ansiedade materna diante do nascimento prematuro se encontram diretamente relacionados a um enfrentamento pouco adaptativo dessa situação. Além disso, mães ansiosas tendem a demonstrar excessiva responsividade e elevada intrusividade, prejudicando as interações com o bebê (Cantilino \& cols., 2010; Wilfong \& cols., 1991; Wijnroks, 2000).

Já mães deprimidas, conforme revelam as revisões da literatura empreendidas por Schwengber e Piccinini (2003) e Schmidt e colaboradores (2005), encontram dificuldades para fornecer ao bebê estimulação adequada, o que inviabiliza 0 estabelecimento de uma relação sincrônica e a manutenção de sequências interativas estáveis, quer seja por retraimento, quer seja por intrusividade. 0 bebê, consequentemente, tende a demonstrar uma acentuada desorganização comportamental, cujas características principais são a redução de atividades, a evitação do contato visual e a restrição dos episódios de vocalização, assim como a ocorrência de dificuldades importantes na alimentação e no sono.

Repercussões de tal quadro clínico, como já mencionado, tendem a comprometer os processos normais do desenvolvimento infantil. Carvalho e colaboradores (2001) apontam, inclusive, que fatores de risco psicossociais - dentre os quais se destaca a inadequação da atitude materna em relação ao bebê prematuro - eventualmente são mais determinantes do que fatores de risco biológicos para o surgimento de problemas de comportamento e aprendizagem até os 10 anos de idade. Além disso, a ocorrência de sintomas clinicamente significativos de ansiedade e/ ou depressão em mães de bebês prematuros é capaz de contribuir para a implementação ou o agravamento de 
outros fatores de risco psicossociais, tais como alto índice de conflito familiar (Vieira \& Linhares, 2011), ou até mesmo potencializar fatores de risco biológicos (Klein \& Linhares, 2006).

O tamanho reduzido da amostra do presente estudo dificulta 0 estabelecimento preciso de um perfil sociodemográfico e clínico das puérperas em situação de risco para o desenvolvimento de sintomas de ansiedade e depressão. Todavia, deve-se mencionar que, tanto entre as mães de bebês prematuros quanto entre as mães de bebês a termo avaliadas, obtiveram escores mais elevados em ambas as subescalas da HAD aquelas que exerciam atividades profissionais que requerem pouca qualificação profissional e, consequentemente, proporcionam baixa remuneração. Esse achado reforça que condições socioeconômicas precárias podem representar um importante fator de risco para o desenvolvimento de transtomos mentais no puerpério (Cantilino \& cols., 2010).

Cumpre assinalar, por fim, que Carvalho e colaboradores (2009) apontam que os grupos de apoio são particularmente proveitosos em face à necessidade de oferecer assistência psicológica às mães de bebês prematuros, uma vez que são capazes de prover suporte social e emocional, contribuindo, assim, para a atenuação de sintomas de ansiedade e depressão. Assumindo essa premissa, foi implementado um grupo de apoio especificamente para mães de bebês prematuros no hospital em questão. Detalhar 0 funcionamento de tal intervenção foge ao escopo do presente estudo, mas é válido mencionar que o mesmo se revelou bastante produtivo.

\section{Considerações finais}

0 presente estudo revela a ocorrência, em uma parcela proporcionalmente maior em comparação com outros estudos, de sintomas clinicamente significativos de ansiedade (75\%) e depressão (50\%) em mães de bebês prematuros. Esse resultado, embora derivado da avaliação de uma amostra de tamanho reduzido, confirma a necessidade de dedicar atenção especial à saúde mental dessa população. 0 presente estudo também atesta a pertinência da HAD para 0 rastreamento de quadros depressivos e ansiosos no contexto hospitalar, sobretudo por ser voltada especificamente à avaliação da sintomatologia psíquica. A utilização do instrumento em questão pode revelar possíveis casos de transtornos do humor que passariam despercebidos pela equipe hospitalar e, assim, subsidiar a implementação das modalidades assistenciais adequadas.

0 presente estudo tem ainda 0 mérito de ser 0 pioneiro na utilização da HAD junto a puérperas. É preciso esclarecer que tal instrumento não se configura como um teste psicológico, de modo que não é restrito a psicólogos nos termos da legislação vigente e pode ser empregado por qualquer profissional de saúde devidamente treinado, o que torna patente sua ampla aplicabilidade. Somando-se a isso as demais vantagens da HAD já mencionadas, sugere-se a realização de novos estudos que a incluam no processo de coleta de dados e envolvam amostras ampliadas e representativas de mães de bebês prematuros, particularmente para comparação com mães de bebês a termo saudáveis ou acometidos por doenças específicas.

\section{Referências}

Andrade, L. H. S., \& Gorenstein, C. (2000). Aspectos gerais das escalas de avaliação de ansiedade. Em C. Gorenstein, L. H. S. Andrade \& A. W. Zuardi (Orgs.), Escalas de avaliação dínica em psiquiatria e psicafamaadoja(pp. 139-144). São Paulo: Lemos.

Botega, N. J., Bio, M. R., Zomignani, M. A., Garcia Junior, C., \& Pereira, W. A. B. (1995). Transtomos de humor em enfermaria de clínica médica e validação de escala de medida (HAD) de ansiedade e depressão. Reuista deSaúdePúdica, 29(5), 355-363.

Calil, H. M., \& Pires, M. L. N. (2000). Aspectos gerais das escalas de avaliação de depressão. Em C. Gorenstein, L. H. S. Andrade \& A. W. Zuardi (Orgs.), Escalas de avaliação dínica em psiquiatria e psicofamadoga (pp. 65-69). São Paulo: Lemos.

Cantilino, A., Zambaldi, C. F., Sougey, E. B., \& Rennó Junior, J. (2010). Transtornos psiquiátricos no pósparto. Revista dePsiquiatria Clínica, 37(6), 278-284.

Carvalho, A. E. V., Linhares, M. B. M., \& Martinez, F. E. (2001). História de desenvolvimento e comportamento de crianças nascidas pré-termo e baixo peso (<1.500 g). Psicdoga: Rflexão e Cńtica, 14(1), 1-33.

Carvalho, A. E. V., Linhares, M. B. M., Padovani, F. H. P., \& Martinez, F. E. (2009). Anxiety and depression in mothers of preterm infants and psychological intervention during hospitalization in neonatal ICU. Spanish Jaumal of Psyddogy, 12(1), 161-170.

Castro, M. M. C., Quarantini, L., Batista-Neves, S., Kraychete, D. C., Daltro, C., \& Miranda-Scippa, A. (2006). Validade da Escala Hospitalar de Ansiedade e Depressão em pacientes com dor crônica. Reista Brasilera de Anstesidoga, 56(5), 470-477.

Cox, J., \& Holden, J. (2003). Peinatal mettal health: a guide to the Edinburg Postnatal Depression Scale Londres: Royal College of Psychiatrists Publications.

D algalarrondo, P. (2008). Psicopatdoga e semidoga dos transtomosmentais Porto Alegre: Artmed.

D elfini, A. B. L., Roque, A. P., \& Peres, R. S. (2009). Sintomatologia ansiosa e depressiva em adultos 
hospitalizados: rastreamento em enfermaria clínica. Geais, 2(1), 12-22.

Fiqueira, P., Corrêa, H., Malloy-D iniz, L., \& RomanoSilva, M. A. (2009). Escala de Depressão Pós-natal de Edimburgo para triagem no Sistema Público de Saúde. Revista deSaúdePúdica, 43(Supl. 1), 79-84.

Kennell, J., \& Klaus, M. (1993). Atendimento para os pais de bebês prematuros ou doentes (D. Batista, trad.). Em M. Klaus \& J. Kennell (O rgs.), Paisbebê a formação do apeeg (pp. 170-244). Porto Alegre: Artes Médicas.

Klein, V. C., \& Linhares, M. B. M. (2006). Prematuridade e interação mãe-criança: revisão sistemática da literatura. Psicdoga emEstuda, 11(2), 277-284.

Marcolino, J. A. M., Mathias, L. A. S. T., Piccinini Filho, L., Guaratini, A. A., Suzuki, F. M., \& Alli, L. A. C. (2007). Escala Hospitalar de Ansiedade e Depressão: estudo da validade de critério e da confiabilidade com pacientes no pré-operatório. Reista Brasilera deAnesteidogia, 57(1), 52-62.

Moreira, M. E. L., \& Rodrigues, M. A. (2003). O bebê na UTI: intercorrências e exames mais frequentes. Em M. E. L. Moreira, N. A. Braga \& D. S. Morsch (O rgs.), Quandb a vida comega diferente o bebê e sua família na UTI-Neonatal (pp. 43-50). Rio de Janeiro: Fiocruz.

Padovani, F. H. P., Linhares, M. B. M., Carvalho, A. E. V., Duarte, G., \& Martinez, F. E. (2004). Avaliação de sintomas de ansiedade e depressão em mães de neonatos pré-termo durante e após hospitalização em UTI-Neonatal. Reista Brasileira dePsiquiatria, 26(4), 251-254.

Padovani, F. H. P., Carvalho, A. E. V., D uarte, G., Martinez, F. E., \& Linhares, M. B. M. (2009). Anxiety, dysphoria, and depression symptoms in mothers of preterm infants. Psyddogical Repats 104(2), 667-679.
Pinelli, J. (2000). Effects of family coping and resources on family adjustment and parental stress in the acute phase of the NICU experience. Neonatal Network, 196), 27-37.

Raad, A. J., Cruz, A. M. C., \& Nascimento M. A. (2006). A realidade das mães numa unidade de terapia intensiva neonatal. Psic 7(2), 85-92.

Schmidt, E. B., Picolotto, N. M., \& Müller, M. C. (2005). Depressão pós-parto: fatores de risco e repercussões no desenvolvimento infantil. Psico USF, 10(1), 61-68.

Schwengber, D. D. S., \& Piccinini, C. A. (2003). O impacto da depressão pós-parto para a interação mãe-bebê. Estudos dePsicdogia, 8(3), 403-411.

Selltiz, C., Wrightsman, L. S., \& Cook, S. W. (1987). Dados de observação e de arquivo (M. M. H. D’Oliveira \& M. M. Del Rey, trads.). Em L. H. Kidder (O rg.), Mátodos de pesquisa nas redaçâs sociais (pp. 95-120). São Paulo: EPU.

Vieira, M. E. B., \& Linhares, M. B. M. (2011). D esenvolvimento e qualidade de vida em crianças nascidas pré-termo em idades pré-escolar e escolar. Jomal dePediatria, 87(4), 281-291.

Wijnroks, L. (2000). Maternal recollected anxiety and mother-infant interaction in preterm infants. Infant Mental Health Jaumal, 20(4), 393-409.

Wilfong, E. W., Saylor, C., \& Elksnin, N. (1991). Influences on responsiveness: interactions between mothers and their premature infants. Infant Mental Health Jaumal, 12(1), 31-40.

Zigmond, A., \& Snaith, R. P. (1983). The Hospital Anxiety and Depression Scale. Ada Psydiatrica Scandinavica, 67(6), 361-370.

Reedidbem10/ 12/ 2011 Reformuladb em28/ 08/ 2012 A provadoem08/ 09/ 2012 
Sobre os autores:

Marina de Souza Filho Favaro é psicóloga pelo Centro Universitário de Araraquara (UNIARA).

Rodrigo Sanches Peres é psicólogo, mestre e doutor em Psicologia, especialista em Psicologia Clínica pelo Conselho Federal de Psicologia (CFP), professor do Programa de Pós-Graduação em Psicologia do Instituto de Psicologia da Universidade Federal de Uberlândia (IP-UFU).

Manoel Antônio dos Santos é psicólogo, mestre e doutor em Psicologia Clínica, livre-docente em Psicoterapia Psicanalítica, professor do Programa de Pós-Graduação em Psicologia da Faculdade de Filosofia, Ciências e Letras de Ribeirão Preto da Universidade de São Paulo (FFCLRP-USP). É bolsista de Produtividade em Pesquisa do Conselho Nacional de Desenvolvimento Científico e Tecnológico (CNPq).

Contato com os autores:

Instituto de Psicologia - Universidade Federal de Uberlândia.

Avenida Pará, 1.720, Bloco 2C, Campus Umuarama - CEP 38405-320 - Uberlândia-MG .

E-mail: rodrigosanchesperes@yahoo.com.br 